\title{
PERFECT PYRAMIDS
}

\section{Ralph Heiner BuchHolZ}

This paper discusses rational edged tetrahedra, in 3, 4 and $n$ dimensions, with rational volume. The main results are (i) a proof of the existence of infinitely many tetrahedra with rational edge-lengths, face-areas and volume and (ii) a proof that there exist dimensions for which all regular hypertetrahedra with rational edgelengths have rational hypervolume.

\section{INTRODUCTION}

Define a perfect pyramid to be a tetrahedron with integer edge-lengths, face areas and volume. This definition can be extended to higher and lower dimensions. For example in one dimension any line segment of integer length is a perfect 1-pyramid. In two dimensions any triangle with integer edges and area, such as the $(3,4,5)$ triangle, is obviously a perfect 2-pyramid. Hero's formula for the area, $\Delta$, of a triangle in terms of the edge-lengths is

$$
\Delta=\sqrt{s(s-a)(s-b)(s-c)} \quad \text { where } s=\frac{a+b+c}{2},
$$

which can be rewritten matrix form as follows:

$$
(4 \Delta)^{2}=\left[a^{2} b^{2} c^{2}\right]\left[\begin{array}{rrr}
-1 & 1 & 1 \\
1 & -1 & 1 \\
1 & 1 & -1
\end{array}\right]\left[\begin{array}{l}
a^{2} \\
b^{2} \\
c^{2}
\end{array}\right] .
$$

The complete integer solution of this diophantine equation [2] was first found by Euler. Carmichael's parametric version [1] is

$$
\begin{gathered}
a=n\left(m^{2}+k^{2}\right) \\
b=m\left(n^{2}+k^{2}\right) \\
c=(m+n)\left(m n-k^{2}\right) \\
\Delta=k m n(m+n)\left(m n-k^{2}\right)
\end{gathered}
$$

which produces one member of each similarity class of Heron triangles for any integers $m, n$ and $k$ such that $\operatorname{gcd}(m, n, k)=1, m n>k^{2}>\left(m^{2} n\right) /(2 m+n)$ and $m \geqslant n \geqslant 1$.

Received 30 April 1991

Copyright Clearance Centre, Inc. Serial-fee code: 0004-9729/91 \$A2.00+0.00. 


\section{VOLUME OF A TETRAHEDRON}

Suppose one were to ask for the analogous formula for the volume of a tetrahedron. Consider the tetrahedron in Figure 1. Position the axes so that the coordinates of the four vertices are given by $A:\left(x_{A}, y_{A}, 0\right), B:(0,0,0), C:(a, 0,0)$ and $D:$ $\left(x_{D}, y_{D}, z_{D}\right)$.

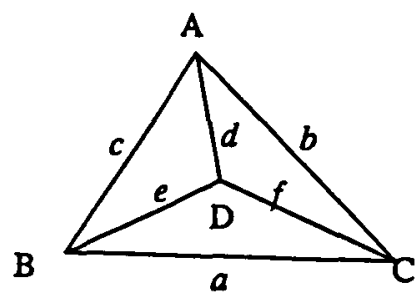

Figure 1

Note that the coordinates of $A$ can be expressed via the cosine rule and area formula as

$$
x_{A}=\frac{a^{2}+c^{2}-b^{2}}{2 a} \text { and } y_{A}=\frac{2 \Delta_{a b c}}{a} \text {. }
$$

The volume of the tetrahedron is

$$
V=\frac{1}{3} z_{D} \Delta_{a b c}
$$

Since the base, $\Delta_{a b c}$, can be expressed in terms of the edge lengths via Heron's formula one need only express the height, $z_{D}$, in terms of the edge lengths. The coordinates of vertex $D$ can be obtained by intersecting three spheres with centres at $A, B$ and $C$ having radii $d, e$ and $f$ respectively. The surfaces of these three spheres are

$$
\begin{gathered}
x^{2}+y^{2}+z^{2}=e^{2} \\
(x-a)^{2}+y^{2}+z^{2}=f^{2} \\
\left(x-x_{A}\right)^{2}+\left(y-y_{A}\right)^{2}+z^{2}=d^{2}
\end{gathered}
$$

and their mutual intersection occurs at $(x, y, z)=\left(x_{D}, y_{D}, \pm z_{D}\right)$. Thus

$$
\begin{aligned}
& x_{D}=\frac{a^{2}+e^{2}-f^{2}}{2 a} \\
& y_{D}=\frac{c^{2}+e^{2}-d^{2}-2 x_{D} x_{A}}{2 y_{A}} \\
& z_{D}^{2}=e^{2}-x_{D}^{2}-y_{D}^{2} .
\end{aligned}
$$


Substituting the expressions for $x_{A}, y_{A}, x_{D}$ and $y_{D}$ into the last equation one obtains

$$
z_{D}^{2}=\frac{16^{2} \Delta_{a e f}^{2} \Delta_{a b c}^{2}-\left[\left(e^{2}-f^{2}-a^{2}\right)\left(a^{2}+b^{2}-c^{2}\right)+2 a^{2}\left(b^{2}+f^{2}-d^{2}\right)\right]^{2}}{64 \Delta_{a b c}^{2} a^{2}} .
$$

Finally, recalling that $9 V^{2}=z_{D}^{2} \Delta_{a b c}^{2}$, this leads to

$$
(24 a V)^{2}=16^{2} \Delta_{a e f}^{2} \Delta_{a b c}^{2}-\left[\left(e^{2}-f^{2}-a^{2}\right)\left(a^{2}+b^{2}-c^{2}\right)+2 a^{2}\left(b^{2}+f^{2}-d^{2}\right)\right]^{2} .
$$

Expanding this gives

$$
\begin{aligned}
(12 V)^{2}= & \left(a^{2}+d^{2}\right)\left(-a^{2} d^{2}+b^{2} e^{2}+c^{2} f^{2}\right)+\left(b^{2}+e^{2}\right)\left(a^{2} d^{2}-b^{2} e^{2}+c^{2} f^{2}\right) \\
& +\left(c^{2}+f^{2}\right)\left(a^{2} d^{2}+b^{2} e^{2}-c^{2} f^{2}\right)-a^{2} b^{2} c^{2}-a^{2} e^{2} f^{2}-b^{2} d^{2} f^{2}-c^{2} d^{2} e^{2}
\end{aligned}
$$

So the volume of a tetrahedron is given by $(12 V)^{2}=X^{t} M X$ where $X=\left[a^{2} b^{2} c^{2} d^{2} e^{2} f^{2}\right]^{t}$ and

$$
M=\left[\begin{array}{cccccc}
-d^{2} & d^{2} & d^{2}+b^{2} & 0 & 0 & 0 \\
e^{2}-c^{2} & -e^{2} & e^{2} & 0 & 0 & 0 \\
f^{2} & f^{2}-a^{2} & -f^{2} & 0 & 0 & 0 \\
0 & 0 & 0 & -a^{2} & a^{2} & a^{2}-b^{2} \\
0 & 0 & 0 & b^{2}-c^{2} & -b^{2} & b^{2} \\
0 & 0 & 0 & c^{2} & c^{2}-a^{2} & -c^{2}
\end{array}\right] .
$$

\section{INTEGER VOLUME TETRAHEDRA}

Using these equations one can search for tetrahedra with integer volumes or even perfect pyramids. Recall that triangles can be categorised into equilateral, isosceles and scalene. Similarly, by equating various numbers of edges a tetrahedron becomes more symmetric and the corresponding problem becomes (one hopes) a little easier to solve. One parameter tetrahedron

(i) $\quad a=b=c=d=e=f$ \#such that $V \in \mathbf{N}$ example

Two parameter tetrahedra

(i) $a=b=c=d=e, f$

(ii) $a=b=c=d, e=f$

(iii) $a=c=d=f, b=e$

(iv) $a=b=c, d=e=f$

(v) $a=d=f, b=c=e$

Three parameter tetrahedra

(i) $\quad a=b=c=d, e, f$

(ii) $a=c=d=f, b, e$

(iii) $a=b=c, d=e, f$

$\begin{array}{cc}0 & - \\ 0 & - \\ \infty & (a, b)=(9,12) \\ 0 & - \\ 0 & -\end{array}$


(iv) $a=f=d, b=c, e$

(v) $\quad a=f=d, b=e, c$

$\begin{array}{cc}? & ? \\ \text { finite } ? & (a, b, c)=(11,15,16) \\ \infty & (a, b, c)=(21,20,11) \\ ? & ? \\ \infty & (a, c, d)=(12,8,9) \\ \infty & (a, b, c)=(12,7,11) \\ ? & ?\end{array}$

(vi) $\quad a=d, b=e, c=f$

(vii) $a=e, b=f, c=d$

(viii) $a=b, c, d=e=f$

(ix) $a=d, b=f, c=e$

(x) $a=e, b=c, d=f$

- 1(i). Substituting the equalities $a=b=c=d=e=f$ into the expression for the volume of a tetrahedron leads to

$$
\begin{gathered}
(12 V)^{2}=2 a^{6}, \\
V=\frac{\sqrt{2} a^{3}}{12} .
\end{gathered}
$$

Clearly if $a \in \mathbf{N}$ then $V \notin \mathbf{N}$. Hence there are no solutions in this case.

- 2(i). Substitution gives

$$
(12 V)^{2}=a^{2} f^{2}\left(3 a^{2}-f^{2}\right) .
$$

Without loss of generality let $\operatorname{gcd}(a, f)=1$ and $a, f, V \in \mathbf{N}$; then there exists an integer, $p$ say, such that

$$
\begin{gathered}
3 a^{2}-f^{2}=p^{2} \\
p^{2}+f^{2}=3 a^{2} \\
p^{2}+f^{2} \equiv 0(\bmod 3)
\end{gathered}
$$

This implies that $3 \mid p$ and $3 \mid f$ and hence $3 \mid a$, which contradicts the assumption of relative primality. Thus no perfect pyramids of this form exist.

- 2(ii). Substitution gives

$$
(12 V)^{2}=a^{2}\left(6 a^{2} e^{2}-\left(a^{2}+e^{2}\right)^{2}\right) .
$$

Without loss of generality, let $\operatorname{gcd}(a, e)=1$ and $a, e, V \in \mathrm{N}$; then there exists an integer, $p$ say, such that

$$
\begin{gathered}
6 a^{2} e^{2}-\left(a^{2}+e^{2}\right)^{2}=p^{2}, \\
p^{2}+\left(a^{2}+e^{2}\right)^{2} \equiv 0(\bmod 3) .
\end{gathered}
$$

This implies that $3 \mid p$ and $3 \mid\left(a^{2}+e^{2}\right)$; hence $3 \mid a$ and $3 \mid e$, clearly a contradiction again, leading to no perfect pyramids of this form. 
- 2(ii). This time

$$
(12 V)^{2}=b^{4}\left(4 a^{2}-2 b^{2}\right) .
$$

If $\operatorname{gcd}(a, b)=1$ and $a, b, V \in \mathbf{N}$, then there exists an integer, $p$ say, such that

$$
4 a^{2}-2 b^{2}=p^{2}
$$

Clearly $p$ must be even, so letting $p=2 P$ gives

$$
2 a^{2}-b^{2}=2 P^{2}
$$

which means that $b$ is even. Hence $b=2 B$ say leads to

$$
a^{2}-P^{2}=2 B^{2} \text {. }
$$

Now $a$ and $P$ must have the same parity so let

to give

$$
\begin{aligned}
& a=Q+S \\
& P=Q-S
\end{aligned}
$$

$$
2 Q S=B^{2} \text {. }
$$

As before $B$ is even so $B=2 D$, say, leads to

$$
Q S=2 D^{2}
$$

Without loss of generality let $Q=2 R$ where $\operatorname{gcd}(R, S)=1$ so that

$$
R S=D^{2}
$$

which has solutions of the form $(R, S, D)=\left(r^{2}, s^{2}, r s\right)$ for some integers $r$ and $s$. Thus back substitution gives an infinite family of integer sided tetrahedra with rational volume namely

$$
\begin{gathered}
a=2 r^{2}+s^{2} \\
b=4 r s \\
V=\frac{8}{3} r^{2} s^{2}\left(2 r^{2}-s^{2}\right) .
\end{gathered}
$$

- 2(iv). Substitution gives $(12 V)^{2}=a^{4}\left(3 d^{2}-a^{2}\right)$ which is the same form as 2(i) and so no solutions exist.

- $2(v)$. Substitution gives

$$
(12 V)^{2}=\left(a^{2}+b^{2}\right)\left(3 a^{2} b^{2}-a^{4}-b^{4}\right) .
$$


If $\operatorname{gcd}(a, b)=1$ and $a, b, V \in \mathrm{N}$ then there exist integers, $m$ and $n$ say, such that

$$
a^{2}+b^{2}=m^{2} \text { and } 3 a^{2} b^{2}-a^{4}-b^{4}=n^{2}
$$

The only solutions to the latter (an elliptic curve of rank 0) occur when $a^{2}=b^{2}$ which makes the former equation insoluble in integers.

- 3(i). Substitution gives $(12 V)^{2}=a^{2}\left(f^{2}\left(2 a^{2}+e^{2}-f^{2}\right)-\left(a^{2}-e^{2}\right)^{2}\right)$. It is unknown whether or not this has solutions but none exist in the range $e, f \leqslant 2 a$, where $a \leqslant 100$.

- 3(ii). Substitution gives $(12 V)^{2}=b^{2} e^{2}\left(4 a^{2}-\left(b^{2}+e^{2}\right)\right)$.

If $\operatorname{gcd}(a, b, e)=1$ and $a, b, e, V \in \mathbf{N}$ then there exists an integer, $n$ say, such that

$$
4 a^{2}-\left(b^{2}+e^{2}\right)=n^{2}
$$

Rearranging leads to

$$
b^{2}+e^{2}+n^{2}=4 a^{2} .
$$

This means that $b, e$ and $n$ must be even numbers so letting $b=2 B, e=2 E, n=2 N$ leads to

$$
B^{2}+E^{2}+n^{2}=a^{2} .
$$

Now $\operatorname{gcd}(a, b, e)=1$ implies that $a$ must be odd. Any number with all its prime divisors equivalent to one modulo 4 has a representation as a sum of two squares. Furthermore it turns out that only numbers of the form $4^{S}(8 m+7)$ can be represented as a sum of four squares (and no fewer). But $a^{2} \equiv 7(\bmod 8)$ is impossible and $a$ is odd and hence $a^{2}$ never takes the form $4^{S}(8 m+7)$. So finally the above equation is soluble for all integers, $a$, in the following set.

$A=\{a: a$ is odd and $\exists$ at least one prime $p$ such that $p \mid a$ implies $p \equiv 3(\bmod 4)\}$. For example $a=3,7,9,11,15, \ldots$ provide the solutions

$$
\begin{aligned}
1^{2}+2^{2}+2^{2} & =3^{2} \\
2^{2}+3^{2}+6^{2} & =7^{2} \\
1^{2}+4^{2}+8^{2} & =9^{2} \\
2^{2}+6^{2}+9^{2} & =11^{2} \\
2^{2}+5^{2}+14^{2} & =15^{2}
\end{aligned}
$$

Since the set $\{p: p$ is prime, $p \equiv 1(\bmod 4)\}$ is infinite so is the set $\{a=3 p$ : $p$ is prime, $p \equiv 1(\bmod 4)\}$ which is a subset of the set $\mathbf{A}$. Thus $|\mathbf{A}|=\infty$ and there is an infinite number of solutions to the last equation. Note that from the second example one obtains $(a, b, e)=(7,4,6)$ which has a volume of 24 . 
- 3(iii). Substitution gives $(12 V)^{2}=a^{2}\left(16 \Delta_{a d f}^{2}-a^{2} f^{2}\right)$ which has no known solutions for $a \leqslant 2 d, f \leqslant 3 d, d \leqslant 100$.

- 3(iv). Substitution gives $(12 V)^{2}=b^{2} e^{2}\left(3 a^{2}-e^{2}\right)-a^{2}\left(a^{2}-b^{2}\right)^{2}$ which has no known solutions for $b \leqslant 2 a, e \leqslant 2 a, a \leqslant 100$.

- $3(v)$. Substitution gives $(12 V)^{2}=b^{4}\left(3 a^{2}-2 b^{2}+c^{2}\right)-a^{2}\left(a^{2}-c^{2}\right)^{2}$ which has the solutions $(a, b, c)=(11,15,16),(12,10,15),(16,10,15),(20,26,39)$ for $b \leqslant 2 a$, $c \leqslant 3 a, a \leqslant 20$.

- 3(vi). Substitution gives

$$
(12 V)^{2}=2\left(a^{2}+b^{2}-c^{2}\right)\left(a^{2}+c^{2}-b^{2}\right)\left(b^{2}+c^{2}-a^{2}\right)
$$

which has four known solutions for $a \leqslant 156, b \leqslant a, c \leqslant a$. These are

$$
(a, b, c)=(21,20,11),(72,65,33),(100,91,69),(100,99,21)
$$

It will be shown in the next section that each of these generates an infinite set of solutions.

- 3(vii). Substitution gives

$$
(12 V)^{2}=5 a^{2} b^{2} c^{2}-a^{4} b^{2}-b^{4} c^{2}-c^{4} a^{2}
$$

which has no solutions for $a \leqslant 100, b \leqslant a, c \leqslant a$.

- 3(viii). Substitution gives

$$
(12 V)^{2}=c^{2}\left(4 a^{2} d^{2}-c^{2} d^{2}-a^{4}\right)
$$

which has one solution for $a \leqslant 2 d, c \leqslant 2 d, d \leqslant 10$, namely $(a, c, d)=(12,8,9)$. From this one can generate an infinite set of solutions.

Let $a=(4 / 3) d$ to give

$$
\begin{gathered}
Q^{2}=320 d^{2}-9^{2} c^{2} ; \\
\text { hence } \\
Q^{2}+K^{2}=5 D^{2}
\end{gathered}
$$

where $K=9 c, D=8 d, Q=(108 V) /(c d)$. This equation has a parametric solution:

$$
\begin{aligned}
& Q=2\left(5 q^{2}-p^{2}\right) \\
& K=p^{2}-10 p q+5 q^{2} \\
& D=p^{2}-2 p q+5 q^{2} .
\end{aligned}
$$


Whence a set of integer volume pyramids is given by

$$
\begin{aligned}
& g a=12\left(p^{2}-2 p q+5 q^{2}\right) \\
& g c=8\left(p^{2}-10 p q+5 q^{2}\right) \\
& g d=9\left(p^{2}-2 p q+5 q^{2}\right)
\end{aligned}
$$

and $g$ is the gcd of the three right hand sides.

- 3 (ix). Substitution gives $(12 V)^{2}=a^{2}\left(16 \Delta_{a b c}^{2}-a^{4}\right)$ which has three solutions for $a \leqslant 2 c, b \leqslant 3 c, c \leqslant 35$, namely $(a, b, c)=(12,7,11),(28,15,27),(36,19,35)$ from which an infinite set of solutions can be obtained. Rewriting the area using Heron's formula gives

$$
\begin{aligned}
& (12 V)^{2}=2 a^{2} b^{2}+2 b^{2} c^{2}+2 a^{2} c^{2}-2 a^{4}-b^{4}-c^{4} \\
& (12 V)^{2}=2 a^{2}\left(b^{2}+c^{2}-a^{2}\right)-(b-c)^{2}(b+c)^{2} .
\end{aligned}
$$

So let $(c-b) / a=(11-7) /(12)=1 / 3$ or $a=3(c-b)$ and $Q=(12 V) /(c-b)$ to give

$$
\begin{gathered}
18\left(b^{2}+c^{2}-9(b-c)^{2}\right)-(b+c)^{2}=Q^{2} \\
322 b c-145 b^{2}-145 c^{2}=Q^{2} \\
(63 b-39 c)^{2}-34(11 b-7 c)^{2}=Q^{2}
\end{gathered}
$$

Letting $B=63 b-39 c$ and $C=11 b-7 c$ leads to the equation

$$
Q^{2}+34 C^{2}=B^{2}
$$

which has the solution

$$
\begin{aligned}
& Q=p^{2}-34 q^{2} \\
& C=2 p q \\
& B=p^{2}+34 q^{2}
\end{aligned}
$$

Substituting this parametrisation into the appropriate equations above and solving for $a, b$ and $c$ leads to

$$
\begin{aligned}
& g a=12 p^{2}-144 p q+408 q^{2} \\
& g c=7 p^{2}-78 p q+238 q^{2} \\
& g d=11 p^{2}-126 p q+374 q^{2}
\end{aligned}
$$

where $g$ is the gcd of the three right hand sides.

- $3(\mathrm{x})$. Substitution gives $(12 V)^{2}=5 a^{2} b^{2} d^{2}-a^{2} b^{4}-a^{4} d^{2}-b^{2} d^{4}$ which has no solutions for $a \leqslant 3 d, b \leqslant 2 d, d \leqslant 100$. 


\section{Perfect pyramids}

Having thus far found many different (though by no means all) sets of tetrahedra with integer volume one can return to the problem of searching for perfect pyramids.

A brute force search is very slow to uncover any - in fact the tetrahedron

$$
(a, b, c, d, e, f)=(117,80,53,52,51,84)
$$

is the only perfect pyramid with $(a, b, c, d, e, f) \leqslant 156$. The volume is 18144 while the face areas are $\Delta_{a b c}=1800, \Delta_{a e f}=1890, \Delta_{b d f}=2016, \Delta_{c d e}=1170$.

Next, one can consider the restrictions to one, two and three parameter tetrahedra. Since no one-parameter tetrahedron can have integer volume there can be no perfect pyramids of this form.

Similarly, for two-parameter tetrahedra one need only consider case (iii). Here each face is a triangle with sides $(a, a, b)=\left(2 r^{2}+s^{2}, 2 r^{2}+s^{2}, 4 r s\right)$. Thus the length, $h$, of the altitude to the side $b$ is given by Pythagoras' theorem as

$$
\begin{aligned}
& h^{2}=a^{2}-(b / 2)^{2} \\
& h^{2}=\left(2 r^{2}+s^{2}\right)^{2}-(2 r s)^{2} \\
& h^{2}=4 r^{4}+s^{4}
\end{aligned}
$$

which has no solutions in positive integers.

A more profitable search can be made by considering the three-parameter tetrahedra. For cases (i) and (iii) the bases are equilateral triangles which cannot have integer area. This immediately precludes the possibility of perfect pyramids of this form.

However, consider case (vi). If the base $(a, b, c)$ is a Heron triangle then all four face areas are integral (since they are all identical). Using Carmichael's parametrisation for Heron triangles one can quickly discover the five pyramids of Table 1 . Note that the third example here has been discovered previously [4] as a solution to problem D22 in Guy's Unsolved Problems in Number Theory [3].

Table 1 - Perfect Pyramids of form $(a, b, c, a, b, c)$

\begin{tabular}{|c|c|c|c|c|c|c|}
\hline$m$ & $n$ & $k$ & $a$ & $b$ & $c$ & $V$ \\
\hline 21 & 20 & 15 & 888 & 875 & 533 & 37608480 \\
\hline 39 & 34 & 26 & 2873 & 2748 & 1825 & 1355172000 \\
\hline 39 & 35 & 25 & 203 & 195 & 148 & 611520 \\
\hline 58 & 33 & 30 & 1804 & 1479 & 1183 & 214582368 \\
\hline 77 & 68 & 44 & 2431 & 2296 & 2175 & 1403038560 \\
\hline
\end{tabular}


THEOREM 1. There exists an infinite number of perfect pyramids with edges of the form $(a, b, c, a, b, c)$.

ProOF: First recall that the volume of a tetrahedron in this case is given by

$$
(12 V)^{2}=2\left(a^{2}+b^{2}-c^{2}\right)\left(a^{2}+c^{2}-b^{2}\right)\left(b^{2}+c^{2}-a^{2}\right)
$$

Now replacing $a, b$ and $c$ via Carmichael's parametrisation first gives

$$
\begin{aligned}
& a^{2}+b^{2}-c^{2}=2 m n\left[k^{2}(m+n)^{2}-\left(m n-k^{2}\right)^{2}\right] \\
& a^{2}+c^{2}-b^{2}=2 n(m+n)\left(m n-k^{2}\right)\left(m^{2}-k^{2}\right) \\
& b^{2}+c^{2}-a^{2}=2 m(m+n)\left(m n-k^{2}\right)\left(n^{2}-k^{2}\right)
\end{aligned}
$$

and hence one obtains

$$
(12 V)^{2}=\left[4 m n(m+n)\left(m n-k^{2}\right)\right]^{2}\left[k^{2}(m+n)^{2}-\left(m n-k^{2}\right)^{2}\right]\left(m^{2}-k^{2}\right)\left(n^{2}-k^{2}\right)
$$

So there exists an integer, $v$ say, such that

$$
v^{2}=\left[k^{2}(m+n)^{2}-\left(m n-k^{2}\right)^{2}\right]\left(m^{2}-k^{2}\right)\left(n^{2}-k^{2}\right) \text {. }
$$

Now dividing through by $k^{8}$ and setting $Z=v / k^{4}, x=m / k, y=n / k$ leads to

$$
Z^{2}=(x+y-x y+1)(x+y+x y-1)(x-1)(x+1)(y-1)(y+1) .
$$

Notice that this is a quartic in $x$ and $y$ and it has rational solutions corresponding to the perfect pyramids in Table 1 , one of which is $(x, y, Z)=(7 / 5,4 / 3,56 / 25)$. The aim now is to show that this solution generates an infinite number of solutions by transforming the equation to an elliptic curve of rank $\geqslant 1$.

Let $y=4 / 3$ and $Z=z / 9$ to give

$$
z^{2}=7(-x+7)(7 x+1)(x-1)(x+1)
$$

which has a rational point $\left(x_{1}, z_{1}\right)=(7 / 5,504 / 25)$. Before transforming this it is first useful to obtain a second rational point on the quartic curve by a method analogous to the tangent-chord process.

Note that the parabola $z_{p}=a_{p} x^{2}+b_{p} x+c_{p}$ where $a_{p}=(-13057) /\left(2^{2} \cdot 3^{4} \cdot 7\right)$, $b_{p}=(40705) /\left(2 \cdot 3^{4} \cdot 5\right), c_{p}=(-12607) /\left(2^{2} \cdot 3^{4}\right)$ meets the quartic curve $(2)$ at the point $\left(x_{1}, z_{1}\right)=(7 / 5,504 / 25)$ such that $z_{p}^{\prime}=z^{\prime}$ and $z_{p}^{\prime \prime}=z^{\prime \prime}$. Hence intersecting this quadratic with equation (2) leads to another quartic equation, $g(x)$ say, which has, by its construction, a root of multiplicity 3 at $x=7 / 5$ and a second root which corresponds to a second rational point on equation (2); see Figure 2. 
Thus equating coefficients of powers of $x$ in

$$
g(x)=z^{2}-z_{p}^{2}=(x-7 / 5)^{3}\left(P_{2} x-Q_{2}\right)=0
$$

leads to $P_{2}=\left(-5^{3} \cdot 677 \cdot 4993\right) /\left(2^{4} \cdot 3^{8} \cdot 7^{2}\right), Q_{2}=\left(-5^{3} \cdot 3348577\right) /\left(2^{4} \cdot 3^{8} \cdot 7\right)$ and so the second rational point on $(2)$ is $\left(x_{2}, z_{2}\right)$ where

$$
x_{2}=\frac{Q_{2}}{P_{2}}=\frac{(7 \cdot 3348577)}{(677 \cdot 4993)}, z_{2}=\frac{\left(2^{3} \cdot 3^{3} \cdot 5 \cdot 7 \cdot 13 \cdot 23 \cdot 89 \cdot 587 \cdot 3167\right)}{\left(677^{2} \cdot 4993^{2}\right)}
$$

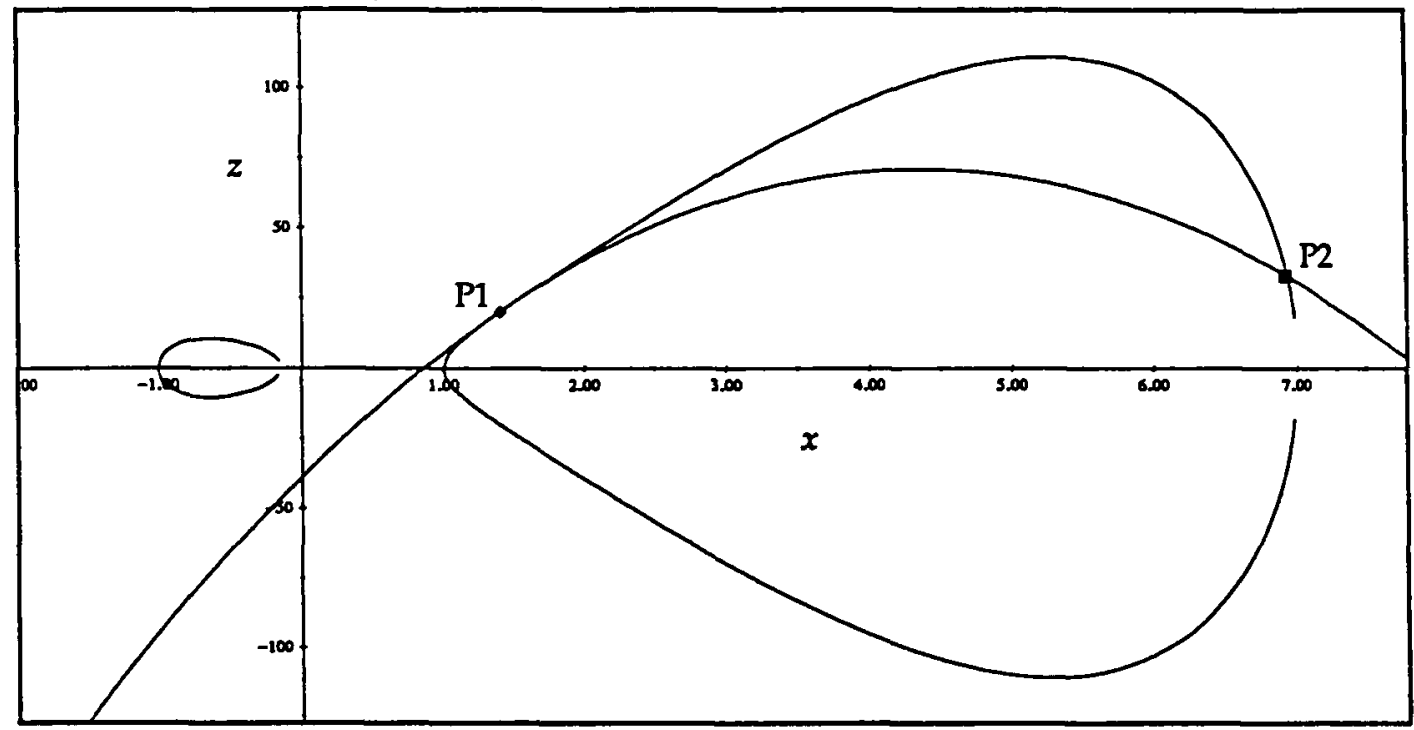

Figure 2

Transform the quartic curve (2) so that the leading term becomes a positive rational square by setting $x=(7 X+1) /(5 X)$ and $z=W /\left(5^{2} X^{2}\right)$. Hence equation (2) becomes

$$
W^{2}=7(28 X-1)(54 X+7)(12 X+1)(2 X+1) \text {. }
$$

Notice that the rational point at $\left(x_{1}, z_{1}\right)=(7 / 5,504 / 25)$ has been transformed to $X=\infty$ which is why the second rational point was required. Now equation (3) can be transformed into a monic quartic equation lacking the cubic term by setting

$$
X=\frac{u-256}{2^{3} \cdot 3^{3} \cdot 7}, \quad W=\frac{w}{2^{3} \cdot 3^{4} \cdot 7} .
$$

Hence

$$
w^{2}=(u-310)(u-60)(u-130)(u+500)
$$

or

$$
w^{2}=u^{4}-6 \cdot 30550 u^{2}+4 \cdot 7733000 u-1209000000 .
$$


Applying Mordell's transformation, namely, $2 u=(t+q) /(s-p)$ and $w=2 s-$ $u^{2}+p$ where $p=30550, q=-7733000$ and $r=-1209000000$ leads to the elliptic curve

$$
t^{2}=4 s^{3}-g_{2} s+g_{3}
$$

where $g_{2}=3 p^{2}+r=1590907500$ and $g_{3}=q^{2}+p r-p^{3}=-5648052375000$. Now since four divides the right hand side of equation (5) we conclude that $t$ must be even, so letting $t=2 T$ leads to

$$
T^{2}=s^{3}-397726875 s-2 \cdot 5^{3} \cdot 5648052375
$$

that is

$$
T^{2}=(s+17850)(s+3675)(s-21525) .
$$

The rational point $\left(x_{2}, z_{2}\right)$ on equation (2) can be transformed into a rational point on equation (6), namely $\left(s_{1}, T_{1}\right)$ where

$$
s_{1}=\frac{5^{2} \cdot 13 \cdot 416834008069}{2^{6} \cdot 11^{2} \cdot 19^{2} \cdot 37^{2}} \text { and } T_{1}=\frac{78688321296560732005909000}{\left(2^{6} \cdot 11^{2} \cdot 19^{2} \cdot 37^{2}\right)^{2}} .
$$

Since $s_{1}$ and $T_{1}$ are not integers then by a theorem of Lutz and Nagel (see [5], p.211), the point $\left(s_{1}, T_{1}\right)$ must have infinite order. Thus equation (6) has an infinite number of rational solutions which, in turn, implies that equation (2) has an infinite number of rational solutions.

The final hurdle is the transformation of the triangle inequalities since only solutions satisfying $m n>k^{2}>\left(m^{2} n\right) /(2 m+n)$ and $m \geqslant n \geqslant 1$ lead to valid perfect pyramids in equation (1). Now

$$
\begin{aligned}
& m n>k^{2} \Rightarrow x y>1 \quad \Rightarrow \quad x>\frac{3}{4} \\
& m>n \quad \Rightarrow \quad x>y \quad \Rightarrow \quad x>\frac{4}{3} \\
& k^{2}>\frac{m^{2} n}{2 m+n} \quad \Rightarrow \quad 1>\frac{x^{2} y}{2 x+y} \quad \Rightarrow \quad-\frac{1}{2}<x<2 \text {. }
\end{aligned}
$$

So the rational points $(x, z)$ on equation (2) which correspond to perfect pyramids are those for which $4 / 3<x<2$ and $(7 \sqrt{17.31}) / 9<z<15 \sqrt{17}$. (In particular the second rational point, $\left(x_{2}, z_{2}\right)$, found above, does not lie in this range and so does not produce a new perfect pyramid.) Continuing the transformation of the triangle inequalities leads ultimately to the corresponding inequality $s>46027+4900 \sqrt{17 \cdot 31}>127963$ for rational points on the elliptic curve (6). Since equation (6) has an infinite number of 
rational points $(s, T)$ satisfying $s>r$ for any constant $r$, one concludes that equation (1) has an infinite number of solutions corresponding to perfect pyramids.

Notice that this also provides an infinite number of solutions for case 3(vi) in Section 3.

Searches for perfect pyramids of the form $3(v)$, (viii) and (ix) have so far failed to turn up any examples.

\section{INTEGER VOLUME SIMPLEX}

For a four dimensional tetrahedron, often called a simplex, (see Figure 3) one can use a similar technique to express the hypervolume, $\mathcal{V}$, in terms of the edge lengths.

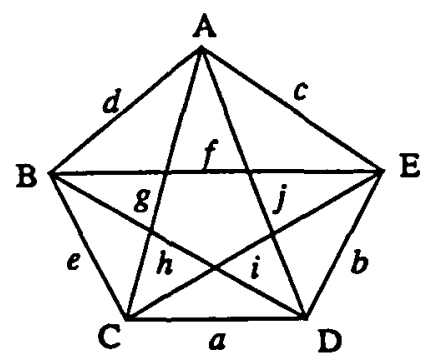

Figure 3

First note that

$$
\nu_{\mathrm{ABCDE}}=\frac{1}{4} V_{\mathrm{ABCD}} w_{\mathrm{E}}
$$

where $w_{\mathrm{E}}$ is the perpendicular height of the point $\mathrm{E}$ above the tetrahedron (ABCD). The volume of the base can be expressed in terms of the edge lengths using the expression in Section 2. So preceeding as before one need only find $w_{E}$ in terms of the edge lengths. Intersecting the four hyperspheres with centres at A, B, C, D and radii $c, f, i, b$ respectively one obtains the hypervolume of a simplex, namely,

$$
(96 \mathcal{V})^{2}=X^{t} M X
$$

where $X=\left[a^{2} b^{2} c^{2} d^{2} e^{2} f^{2} g^{2} h^{2} i^{2} j^{2}\right]^{t}, M=\left(\begin{array}{cc}P & Q \\ R & S\end{array}\right)$ and

$$
\begin{aligned}
& P= \\
& {\left[\begin{array}{ccccc}
-d^{4}-16 \Delta_{c d f}^{2} & 2 d^{2}\left(f^{2}+g^{2}\right) & -2\left(b^{2} g^{2}+i^{2} j^{2}\right) & 2 j^{2}\left(i^{2}+f^{2}\right) & 2\left(d^{2} b^{2}+j^{2} f^{2}\right) \\
2\left(e^{2} c^{2}+f^{2} g^{2}\right) & -e^{4}-16 \Delta_{d e g}^{2} & 2 e^{2}\left(g^{2}+h^{2}\right) & -2\left(c^{2} h^{2}+f^{2} j^{2}\right) & 2 f^{2}\left(j^{2}+g^{2}\right) \\
2 g^{2}\left(f^{2}+h^{2}\right) & 2\left(a^{2} d^{2}+g^{2} h^{2}\right) & -a^{4}-16 \Delta_{e a h}^{2} & 2 a^{2}\left(h^{2}+i^{2}\right) & -2\left(d^{2} i^{2}+g^{2} f^{2}\right) \\
-2\left(e^{2} j^{2}+h^{2} g^{2}\right) & 2 h^{2}\left(g^{2}+i^{2}\right) & 2\left(b^{2} e^{2}+h^{2} i^{2}\right) & -b^{4}-16 \Delta_{a b i}^{2} & 2 b^{2}\left(i^{2}+j^{2}\right) \\
2 c^{2}\left(j^{2}+f^{2}\right) & -2\left(a^{2} f^{2}+i^{2} h^{2}\right) & 2 i^{2}\left(h^{2}+j^{2}\right) & 2\left(c^{2} a^{2}+i^{2} j^{2}\right) & -c^{4}-16 \Delta_{b c j}^{2}
\end{array}\right]}
\end{aligned}
$$




$$
\begin{aligned}
& Q=\left[\begin{array}{ccccc}
-4 c^{2} d^{2} & 0 & 0 & 0 & 0 \\
0 & -4 d^{2} e^{2} & 0 & 0 & 0 \\
0 & 0 & -4 e^{2} a^{2} & 0 & 0 \\
0 & 0 & 0 & -4 a^{2} b^{2} & 0 \\
0 & 0 & 0 & 0 & 4 b^{2} c^{2}
\end{array}\right] \\
& R=\left[\begin{array}{ccccc}
-4 j^{2} g^{2} & 0 & 0 & 0 & 0 \\
0 & -4 f^{2} h^{2} & 0 & 0 & 0 \\
0 & 0 & -4 g^{2} i^{2} & 0 & 0 \\
0 & 0 & 0 & -4 h^{2} j^{2} & 0 \\
0 & 0 & 0 & 0 & -4 i^{2} j^{2}
\end{array}\right]
\end{aligned}
$$

$S=$

$$
\left[\begin{array}{ccccc}
-a^{4}-j^{4}-16 \Delta_{a j g}^{2} & 2 d^{2}\left(a^{2}+b^{2}\right) & 2 g^{2}\left(a^{2}+c^{2}\right) & 2\left(g^{2} h^{2}+d^{2} a^{2}\right) & -2 c^{2} h^{2} \\
-2 d^{2} i^{2} & -b^{4}-f^{4}-16 \Delta_{b f h}^{2} & 2 e^{2}\left(b^{2}+c^{2}\right) & 2 h^{2}\left(b^{2}+d^{2}\right) & 2\left(h^{2} i^{2}+e^{2} b^{2}\right) \\
2\left(i^{2} j^{2}+a^{2} c^{2}\right) & -2 e^{2} j^{2} & -c^{4}-g^{4}-16 \Delta_{c g i}^{2} & 2 a^{2}\left(c^{2}+d^{2}\right) & 2 i^{2}\left(c^{2}+e^{2}\right) \\
2 j^{2}\left(d^{2}+a^{2}\right) & 2\left(j^{2} f^{2}+b^{2} d^{2}\right) & -2 a^{2} f^{2} & -d^{4}-h^{4}-16 \Delta_{d h j}^{2} & 2 b^{2}\left(d^{2}+e^{2}\right) \\
2 c^{2}\left(e^{2}+a^{2}\right) & 2 f^{2}\left(e^{2}+b^{2}\right) & 2\left(f^{2} g^{2}+c^{2} e^{2}\right) & -2 b^{2} g^{2} & -e^{4}-i^{4}-16 \Delta_{e i f}^{2}
\end{array}\right]
$$

Using this one readily discovers that the simplex with edge-lengths given by

$$
(a, b, c, d, e, f, g, h, i, j)=(3,1,2,1,3,2,3,2,3,2)
$$

has a rational hypervolume of $21 / 96$. Thus scaling each edge up by a factor of 32 leads to a simplex with an integer hypervolume of 229376 .

Since any simplex has 10 edges, 10 faces, 5 volumes and 1 hypervolume, the occurrence of a perfect 4-pyramid, which requires all these quantities to be integral, is probably a rarity at best.

\section{REgular N-SimpleX}

Define an $n$-simplex to be the $n$ dimensional analogue of a tetrahedron, that is, $n+1$ vertices connected by $n(n+1) / 2$ edges such that no set of $m$ vertices (and corresponding edges) can be embedded in an $m-2$ dimensional subspace. Some associated quantities of an $n$-simplex are

$V_{n}$ : hypervolume of a regular $n$-simplex with edge length $a$;

$R_{n}$ : circumhypersphere radius of a regular $n$-simplex with edge length $a$;

$r_{n}$ : inhypersphere radius of a regular $n$-simplex with edge length $a$;

$h_{n}$ : distance from any vertex to circumcentre of the opposite regular $(n-1)$ simplex. 
The recurrence relations relating these quantities are

$$
\begin{aligned}
& h_{n}^{2}+R_{n-1}^{2}=a^{2} \\
& V_{n}=\frac{1}{n} V_{n-1} h_{n} \\
& V_{n}=\frac{n+1}{n} V_{n-1} r_{n} \\
& R_{n}^{2}=r_{n}^{2}+R_{n-1}^{2} .
\end{aligned}
$$

Since $V_{1}=h_{1}=1$ and $R_{1}=r_{1}=1 / 2$, one can use induction to prove that the explicit equations for the quantities $V_{n}, R_{n}, r_{n}, h_{n}$ of an $n$-dimensional simplex are given by

$$
\begin{aligned}
V_{n} & =\frac{a^{n}}{n !} \sqrt{\frac{n+1}{2^{n}}}, \\
h_{n} & =\frac{a}{\sqrt{2}} \sqrt{\frac{n+1}{n}}, \\
R_{n} & =\frac{a}{\sqrt{2}} \sqrt{\frac{n}{n+1}}, \\
r_{n} & =\frac{a}{\sqrt{2 n(n+1)}} .
\end{aligned}
$$

TheOREM 2. Any regular, rational-edged, $n$-simplex has rational hypervolume if and only if $n$ takes the form $4 d(d+1)$ or $2 d^{2}-1$.

PROoF: If $n=2 m$ then

$$
V_{2 m}=\frac{a^{2 m} \sqrt{2 m+1}}{(2 m) ! 2^{m}}
$$

which is rational (for rational $a$ ) whenever $2 m+1=d^{2}$ say. Since $d$ must be odd let $d=2 D+1$ so that $n=4 D(D+1)$ gives

$$
V_{4 D(D+1)}=\frac{(2 D+1) a^{4 D(D+1)}}{(4 D(D+1)) ! 2^{2 D(D+1)}} .
$$

So for $n=1,7,17,31,49, \ldots$ dimensional spaces any rational edged $n$-simplex has rational hypervolume.

If $n=2 m+1$ then

$$
V_{2 m+1}=\frac{a^{2 m+1} \sqrt{m+1}}{(2 m+1) ! 2^{m}}
$$

which is rational (for rational $a$ ) whenever $m+1=d^{2}$ say. Thus $n=2 d^{2}-1$ leads to

$$
V_{2 d^{2}-1}=\frac{d a^{2 d^{2}-1}}{\left(2 d^{2}-1\right) ! 2^{d^{2}-1}} \text {. }
$$

So for $n=8,24,48,80, \ldots$ dimensional spaces any rational edged $n$-simplex has rational hypervolume. 


\section{REFERENCES}

[1] R.D. Carmichael, The theory of numbers and diophantine analysis (Dover, 1952).

[2] L.E. Dickson, History of the theory of numbers 2 (Chelsea, 1952).

[3] R.K. Guy, Unsolved problems in number theory (Springer-Verlag, Berlin, Heidelberg, New York, 1981).

[4] R.K. Guy, private communication.

[5] J.H. Silverman, The arithmetric of elliptic curves (Springer-Verlag, Berlin, Heidelberg, New York, 1986).

Department of Mathematics

University of Newcastle

New South Wales 2308

Australia 